\title{
On the probability of occurrence of rogue waves
}

\author{
E. M. Bitner-Gregersen ${ }^{1}$ and A. Toffoli ${ }^{2}$ \\ ${ }^{1}$ Det Norske Veritas, Veritasveien 1, 1322 Høvik, Norway \\ ${ }^{2}$ Swinburne University of Technology, P.O. Box 218, Hawthorn, 3122 Vic., Australia \\ Correspondence to: E. M. Bitner-Gregersen (elzbieta.bitner-gregersen@dnv.com)
}

Received: 15 September 2011 - Revised: 31 January 2012 - Accepted: 5 February 2012 - Published: 23 March 2012

\begin{abstract}
A number of extreme and rogue wave studies have been conducted theoretically, numerically, experimentally and based on field data in the last years, which have significantly advanced our knowledge of ocean waves. So far, however, consensus on the probability of occurrence of rogue waves has not been achieved. The present investigation is addressing this topic from the perspective of design needs. Probability of occurrence of extreme and rogue wave crests in deep water is here discussed based on higher order time simulations, experiments and hindcast data. Focus is given to occurrence of rogue waves in high sea states.
\end{abstract}

\section{Introduction}

Waves of exceptional height and/or abnormal shape, often called freak or rogue waves, have been subjected to much attention in the last decade. Such extreme events are believed to have caused a number of marine accidents with subsequent pollution of large coastal areas, ship damage and human casualties (see, for example, Kharif and Pelinovsky, 2003; Dysthe et al., 2008). Recent hurricanes in the Gulf of Mexico have particularly confirmed the vulnerability of marine infrastructures to extreme waves and highlighted that improvements are needed to reduce the risk associated with these events.

The knowledge of extreme and rogue waves have significantly advanced recently. A number of extreme and rogue wave studies have been conducted theoretically, numerically, experimentally and based on field data (see, e.g. Kharif et al., 2009; Gramstad and Trulsen, 2007; Mori et al., 2002; Onorato et al., 2005, 2009b; Socquet-Juglard et al., 2005; Tamura et al., 2009; Toffoli et al., 2007, 2010; Waseda et al., 2009, 2011, among others). In particular, it has been demonstrated that the contribution of high order nonlinear mechanisms such as the modulational instability of uniform wave packets (see, e.g. Zakharov and Ostrovsky, 2009; Osborne, 2010, for a review) may give rise to substantially higher waves than predicted by common second order wave models (Onorato et al., 2006; Socquet-Juglard et al., 2005; Toffoli et al., 2008b).

The recent Rogue Waves 2008 Workshop (Olagnon and Prevosto, 2008) has brought further insight into rogue waves and their impact on marine structures, underlining the importance of more detailed investigations of meteorological and oceanographic conditions in which extreme and rogue waves occur. The risk associated with such extreme events has also attracted the attention of the shipping and offshore industry, which has recently initiated two international research projects: the JIP project CresT (Cooperative Research on Extreme Seas and their impacT, http://www.marin.nl/web/JIPs-Networks/Public/CresT.htm) with its second phase ShortCresT, and the EU project EXTREME SEAS (http://www.mar.ist.utl.pt/extremeseas/).

Despite recent achievements, consensus on the definition of rogue waves and particularly their probability of occurrence has not been reached. Such consensus would bring further insight to physical features of extreme and rogue waves and characteristic parameters describing them, which is essential for the marine industry. In particular, it is mandatory for evaluation of possible revision of offshore standards and classification society rules, which currently do not include rogue waves explicitly. The traditional format of Classification Societies Rules is mainly prescriptive, without a transparent link to an overall safety objective. The International Maritime Organization (IMO, 1997, 2001) has developed guidelines for use of the Formal Safety Assessment (FSA) methodology in rule development, which will provide riskbased goal-oriented regulations. The FSA methodology is applied also in offshore standard development. It will be used when implementation of rogue waves in design practice 
is considered. Risk is defined in FSA as a product of probability of occurrence of a hazard (e.g. a rogue event) multiplied by its consequence estimated usually as a monetary value (Skjong and Bitner-Gregersen, 2002).

In the design process marine structural strength is evaluated for a given return period (i.e. a time period during which a hazard that can endanger the structure integrity appears not more than once). Ship structural strength and ship stability are calculated, following international standards, in extreme events with an occurrence of once in every $25-\mathrm{yr}$ (Ultimate Limit State, ULS). Offshore structures (including FPSOs: floating production storage and offloading units) follow a different approach to design of ship structures and are designed for the 100-yr return period (ULS). The Norwegian offshore standards (NORSOK, 2007) require that there must be enough space for the wave crest to pass beneath the deck to ensure that a 10000 -yr wave load does not endanger the structure integrity (Accidental Limit State, ALS). But it still lacks guidelines for model predictions of rogue waves and design scenarios to be included in a possible ALS procedure where rogue waves are accounted for. Knowledge about probability of occurrence of rogue waves is necessary for providing a consistent risk-based approach combining new information about extreme and rogue waves in a design perspective. Statistical characteristics of the sea surface like skewness and kurtosis as well as the wave crest distribution will need to be a part of such risk-based approach. Nowadays, the second-order theory-based crest distributions suggested by Forristall (2000) are commonly used in design practice (see DNV, 2010).

The present study attempts to establish the probability of occurrence of extreme and rogue crest height in deep water. The approach is based on new physical understanding of the rogue waves phenomenon, by considering nonlinear wave mechanisms beyond the second order, and statistical considerations of long- and short-term wave statistics. To accomplish this task, higher order time domain simulations of sea surface elevation, laboratory experiments and hindcast data are used. The paper is organized as follows: Sect. 2 discusses the definition of rogue waves as well as their probability of occurrence from the perspective of design needs. Sect. 3 addresses long-term statistics, while Sect. 4 is dedicated to short-term statistics of extreme wave crest and sea state duration. Conclusions are summarized in Sect. 5.

\section{Definition of rogue waves and their probability of occurrence}

Different simplified definitions for rogue waves have been proposed in the literature and are commonly used. Often applied, for example, is the maximum wave height factor $\mathrm{HF}=H_{\max } / H_{\mathrm{s}}>2$ (where $H_{\max }$ is the maximum wave height and $H_{\mathrm{s}}$ is the significant wave height, Rosenthal and Lehner, 2008). Another commonly advocated definition is the criterion based on the maximum crest factor $\mathrm{CF}=c_{\max } / H_{\mathrm{s}}>1.2$ within a 20 -min sea elevation time series (Haver and Andersen, 2000). Tomita and Kawamura (2000) have proposed to use both criterion simulataneously, i.e. HF $>2$ and CF $>1.3$. Guedes Soares et al. (2004), however, argued that the use of the maximum height and crest factor may not be sufficient.

Several authors have pointed out that the kurtosis, i.e. the fourth order moment of the probability density function, can be regarded as a suitable parameter to identify presence of a rogue event in a short-term wave record (e.g. Janssen, 2003; Mori and Janssen, 2006; Mori et al., 2011). It has been verified both theoretically and experimentally that the kurtosis depends on the square of the Benjamin-Feir index (BFI), which is a measure of the relative importance of nonlinearity and dispersion. This index can be defined as $\mathrm{BFI}=\left(k_{\mathrm{p}} H_{\mathrm{s}} / 2\right) /\left(\Delta \omega / \omega_{\mathrm{p}}\right)$, where $k_{\mathrm{p}} H_{\mathrm{s}} / 2$ is the wave steepness $\left(k_{\mathrm{p}}\right.$ is the wavenumber at the spectral peak) and $\Delta \omega / \omega_{\mathrm{p}}$ is the frequency spectral bandwidth ( $\Delta \omega$ is the halfwidth at half-maximum of the spectrum and $\omega_{\mathrm{p}}$ is the spectral peak frequency); see Onorato et al. (2006) for more details on the computation of the Benjamin-Feir index. Random waves are expected to become unstable when $\mathrm{BFI}=\mathrm{O}(1)$, provided the wave field is long crested (i.e. unidirectional wave propagation).

A consensus about a definition of a rogue event will allow the marine industry to reach an agreement about its probability of occurrence. For design purposes, both short- and long-term statistics needs to be considered. The short-term statistical models will provide probability of occurrence of extreme and rogue waves in a given sea state, being of limited duration (e.g. 20-30 min, $1 \mathrm{~h}, 3 \mathrm{~h}$ or $6 \mathrm{~h}$ duration). The long-term statistics, which can cover a time period from 1yr to millennia, will allow determining how often such sea states with extreme and rogue waves will occur within a specific long-term time period. Eventually, both short-term and long-term statistics needs to be combined to provide the total probability of occurrence of, e.g. rogue wave crest, in a required by design time period (a return period).

In design, the probability of extreme wave crest (for a given return period) is calculated by combining the long-term properties of sea state parameters with the conditional shortterm statistics of crests for given sea state parameters (e.g. $H_{\mathrm{s}}$ and $T_{\mathrm{p}}$ ). Long-term extreme values of crest characteristics can be determined by direct calculation of an integral expression combining short-term and long-term statistics, or by estimating the extreme value as a characteristic high value in an extreme sea state condition. These approaches require identification of quantities being of particular interest, e.g. the crest height $c$ and/or the ratio $c_{\mathrm{r}}=c / H_{\mathrm{s}}$. Hereafter, we will refer to the non-dimensional wave crests $c_{\mathrm{r}}$ for convenience.

Pursuing the first analysis approach, the extreme value distribution of crest heights $c$ in a random sea state can be expressed, for example, as 
$F_{\mathrm{EL}}\left(c_{\mathrm{r}}\right)=\int_{H_{\mathrm{s}}} \int_{T_{\mathrm{p}}} F_{\mathrm{ES}}\left(c_{\mathrm{r}} \mid H_{\mathrm{s}}, T_{\mathrm{p}}, \tau\right) f_{H_{\mathrm{s}}, T_{\mathrm{p}}}\left(H_{\mathrm{s}}, T_{\mathrm{p}}\right) d H_{\mathrm{s}} d T_{\mathrm{p}}(1)$

where $f_{H_{\mathrm{s}}, T_{\mathrm{p}}}\left(H_{\mathrm{s}}, T_{\mathrm{p}}\right)$ is the joint probability density of $H_{\mathrm{s}}$ and $T_{\mathrm{p}}$ (assuming $H_{\mathrm{s}}$ and $T_{\mathrm{p}}$ describe satisfactory a sea state; other sea state parameters could be included, if justified, such as the JONSWAP gamma parameter), and $F_{\mathrm{ES}}\left(c_{\mathrm{r}} \mid H_{\mathrm{s}}, T_{\mathrm{p}}, \tau\right)$ is the distribution function for the maximum crest in a given sea state $\left(H_{\mathrm{s}}, T_{\mathrm{p}}\right)$ of duration $\tau$. The required short-term statistics of crest heights need to be provided to carry out these calculations.

Alternatively, a K-yr return period sea state (a design sea state) of a given duration $\tau$ can be estimated (BitnerGregersen, 2003). A common approach is to determine a $\mathrm{K}$-yr $H_{\mathrm{S}}$ level as a fractile of a marginal long-term distribution of significant wave height, combined with adequately selected characteristic values for other sea-state parameters (see, Bitner-Gregersen, 2012). The marginal distribution may represent all sea states or storm peaks only. The approach can be generalized by defining the extreme significant wave height condition in terms of the extreme value distribution of the significant wave height during the interval (annual or lifetime) instead of the deterministic quantile value. The design sea state approximation assumes that the extreme wave crest in the K-yr sea state occurs during the K-yr maximum significant wave height condition. Provided a K-yr sea state, an extreme wave crest is calculated for a given sea state duration $\tau$ as highlighted in Sect. 4.2. Generally, this procedure somewhat underestimates the extreme crests. In order to account for this uncertainty in design practice, a K-yr significant wave height value is often increased by e.g. inflating the marginal significant wave height distribution (see, e.g., Haver and Winterstein, 2009).

The occurrence of rogue waves in a short-term wave record will depend on sea state duration (Bitner-Gregersen, 2003) representing an important parameter for design. Due to randomness of wave surface we can observe rogue waves satisfying the aforementioned definitions in the linear wave model as well as the second-order wave models, if we simulate the water surface elevation long enough. It should be mentioned, however, that the physics described by the linear and second order wave models is different from the one provided by higher order solutions of the water wave problem. Numerical simulations based on the truncated potential Euler equations (see Toffoli et al., 2008a,b; Toffoli and BitnerGregersen, 2011) have indicated that rogue wave crests, e.g. $\mathrm{HF}>2$ and $\mathrm{CF}>1.3$, may occur in a 20-min wave record with a substantially higher probability, as high as $10^{-3}$ and about one order of magnitude higher than second order predictions, if waves are long-crested. Note, however, that the probability is notably decreased to approximately $10^{-4}$ if waves are short crested.

Very limited studies have been carried out aiming at providing probability of occurrence of rogue waves in the ocean. An attempt to establish probability of occurrence of rogue waves by combining short-term and long-term statistics have been carried out by Bitner-Gregersen and Hagen (2004). The probability of occurrence of extreme waves has been calculated using the simplified definition of rogue waves, $c_{\max } / H_{\mathrm{S}}>1.3$, and a second order wave model. The North Sea scatter diagram of significant wave height and spectral peak period was used. The analysis has shown that extreme waves with $c_{\max } / H_{\mathrm{s}}>1.3$ occur with probability corresponding to a return period of about 8 days. They can be observed in low, moderate, as well as high sea states, although they seem to occur more frequently in moderate and low sea states. The results in Bitner-Gregersen and Hagen (2004) should be considered as indicative and site specific and are expected to be somewhat non-conservative as the second order wave model has been used.

Olagnon (2008) has analysed a large short-term wave data set recorded at the Alwyn station in the North Sea. The techniques used to validate applicability of the data for the study were detailed on the grounds of physical limits on the water velocity, accelerations and other qualities. The study has shown that extreme waves are no more frequent than commonly applied in engineering practice statistics would predict.

A number of investigations have also attempted to find relations between individual wave parameters and sea state characteristics identifying occurrence of rogue waves but have not succeeded to find any strong correlation (see, e.g. Olagnon and Magnusson, 2004; Christos and Ewans, 2011a,b). Recently Waseda et al. (2011) used the number of records containing a freak (rogue) wave per certain time window as a proxy to estimating the enhancement of the tail of the probability density function. The authors have tested various definitions of a freak event, using as large threshold as $H_{\max } / H_{\mathrm{s}}>2.4$. Interestingly enough, the authors have found that there is a notable increase of the probability of occurrence of freak waves when the sea level pressure gradient is strengthened and the directional wave spectrum is relatively narrow (directional spread about 30 degrees).

Bascheck and Imai (2011) have suggested that ships predominantly travelling in the North Atlantic (the route from Rotterdam, Netherlands, to New York, USA) will encounter 20-30 rogue waves higher than $11 \mathrm{~m}$ during their service lives of 25-yr. The criterion $H_{\max } / H_{\mathrm{s}}>2.0$ has been used and the conclusions are supported by the analysis of directional wave buoy data, therefore they can be regarded as unconservative.

\section{Long-term statistics}

\subsection{Data}

Met-ocean conditions and sea states in which rogue waves occur are closely related to the mechanism generating them (Kharif and Pelinovsky, 2003). In this respect, the present 


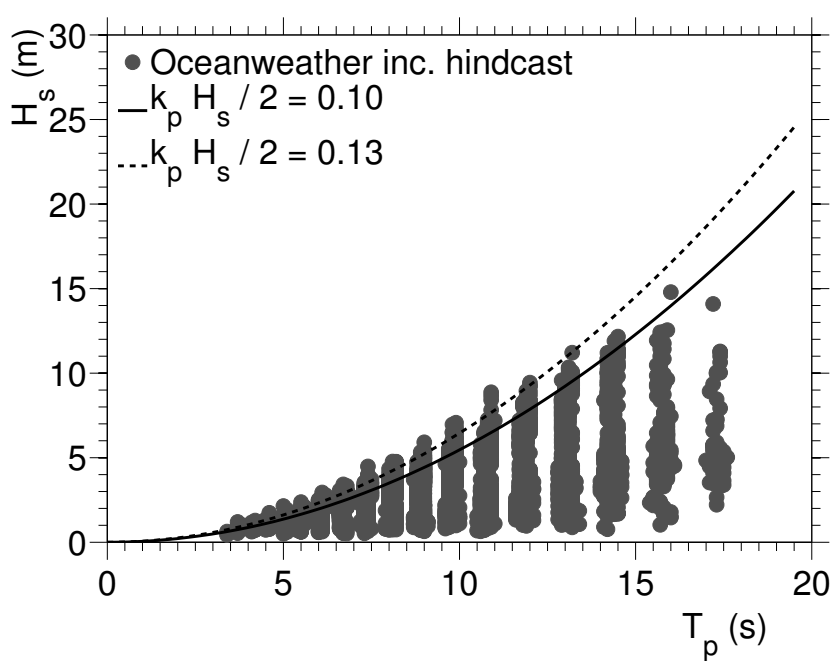

Fig. 1. Scatter diagram of significant wave height $\left(H_{\mathrm{S}}\right)$ and spectral peak period $\left(T_{\mathrm{p}}\right)$ for total seas from Oceanweather Inc. hindcast data; lines of constant spectral steepness $\left(k_{\mathrm{p}} H_{\mathrm{s}} / 2=0.10\right.$ and 0.13$)$ are included.

study only refers to the nonlinear modulational instability of deep water wave trains propagating outside the influence of ocean currents (thus, effects related to wave-current interaction and bottom topography are excluded). We attempt to answer whether sea states prone to rogue waves (e.g. with $\mathrm{BFI}=\mathrm{O}(1))$ can be observed in nature and how often they occurred. To this end, two sets of hindcast data have been used in the analysis. The datasets have been generated for two deep water locations near West of Shetland Islands: the ECMWF reanalysis dataset provided by Meto France and the data generated by the Oceanweather Inc. obtained from Shell. Both locations are in the area being a part of the ship wave design data basis.

The Oceanweather Inc. hindcast data cover the period 1988-1998; wind and wave data are sampled every $3 \mathrm{~h}$. They have been post-processed by Shell using the program APL Waves, developed by the Applied Physics Department of Jonhs Hopkings University. The program divides 3-D spectra (i.e. directional spectra) into separate peaks. The partitioning of the spectrum was performed in order to separate the wind sea from the swell components. The method allows the frequency-direction spectrum to be split into any number of components. Since only bimodal spectra are considered in the present study, components that have been recombined to provide only two components per spectrum (i.e. the wind sea and the total swell).

The ECMWF data cover the period 2001-2009 and are stored at a sampling frequency of $6 \mathrm{~h}$. The wind sea component of wave spectral energy is detected as the part of spectrum where the wind input source term is positive. The components of the wind sea are those which are still under the

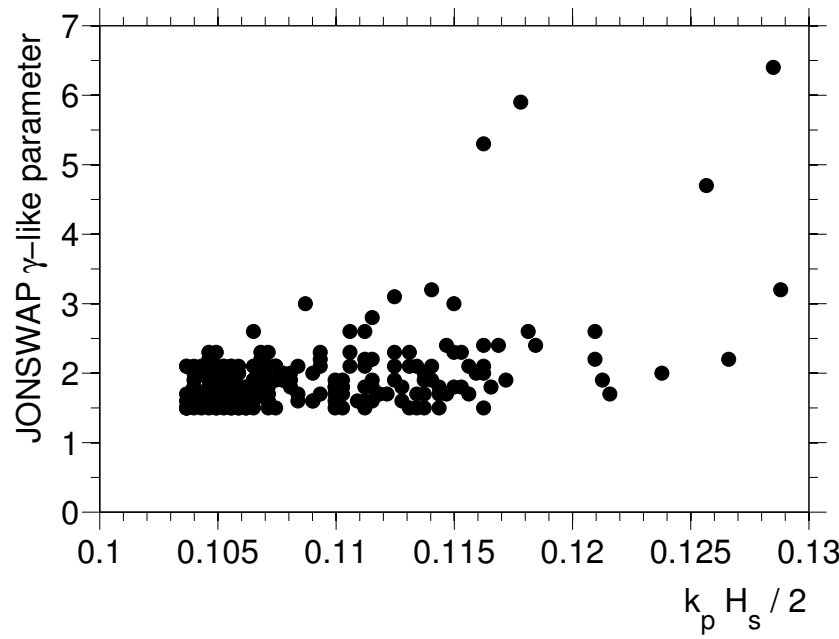

Fig. 2. JONSWAP $\gamma$-like parameter as a function of $k_{\mathrm{p}} H_{\mathrm{s}} / 2$ for wind seas (Oceanweather Inc. hindcast).

influence of the local wind forcing. The remaining part of the spectrum is considered as swell (see, for example, Hauser et al., 2005, for details).

For the present investigation, the following spectral parameters were considered: significant wave height (total sea, wind sea and swell), spectral wave period (total sea, wind sea and swell), wave direction (total sea, wind sea and swell), a JONSWAP $\gamma$-like parameters as well as the mean directional spreading. It is important to note, however, that the accuracy of hindcast is still questioned in the literature (see, e.g. Bitner-Gregersen and Guedes Soares, 2007; BitnerGregersen and de Valk, 2011; Loffredo et al., 2009) and the data used in the analysis are also affected by some uncertainties. However, specification and discussion of these uncertainties is outside the scope of the present study.

\subsection{Analysis}

The two hindcast datasets that are used in the present analysis have been carefully investigated and none outliers have been found. Although the data cover only the 10-yr periods, a reliable 10-yr dataset may provide often good basis for specification of design criteria. Note that extreme values do not increase linearly but exponentially (see Gumbel, 1958). Fits of joint distributions of $H_{\mathrm{s}}$ and $T_{\mathrm{p}}$ as well as $H_{\mathrm{s}}$ and wind speed to the Oceanweather Inc. data can be found in Bitner-Gregersen (2005).

The scatter diagram of the significant wave height and spectral peak period for total sea from the Oceanweather Inc. database is presented in Fig. 1; the lines for constant steepness $k_{\mathrm{p}} H_{\mathrm{s}} / 2=0.10$ and 0.13 are also displayed for reference. These values of steepness have been adopted as they have been proven sufficient to trigger modulational 


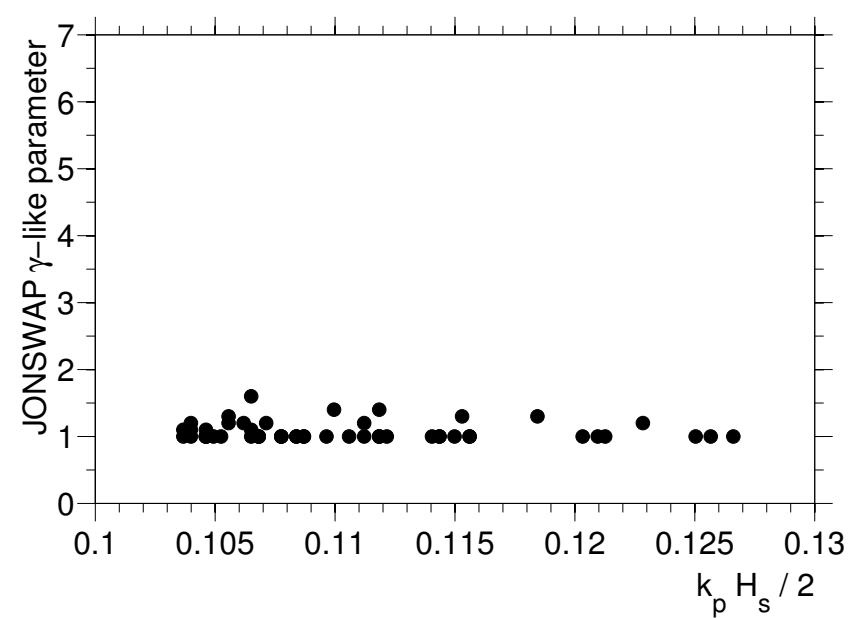

Fig. 3. JONSWAP $\gamma$-like parameter as a function of $k_{\mathrm{p}} H_{\mathrm{s}} / 2$ for swells (Oceanweather Inc. hindcast).

wave instability effects in the laboratories and numerical model tanks (cf. Onorato et al., 2009b; Waseda et al., 2009; Toffoli et al., 2010). Note also that the design standards recommend a limit on sea state steepness expressed as $S_{\mathrm{tp}}=H_{\mathrm{s}} /\left(1.56 T_{\mathrm{p}}^{2}\right)=0.033(\mathrm{DNV}, 2010)$, which corresponds to $k_{\mathrm{p}} H_{\mathrm{s}} / 2=\pi H_{\mathrm{s}} /\left(1.56 T_{\mathrm{p}}^{2}\right) \approx 0.10$. Sea states with steepness equal to or larger than this threshold represent $25 \%$ of the entire database. However, as expected, most of these cases are low or moderated sea states (e.g. $H_{\mathrm{s}}<6 \mathrm{~m}$ ). Approximately $10 \%$ of them, nonetheless, occurred in extreme conditions, where $H_{\mathrm{s}}>6 \mathrm{~m}$. Note also that the highest observed sea state within the 10 -yr time period analysed is characterised by $k_{\mathrm{p}} H_{\mathrm{s}} / 2 \approx 0.13$.

The occurrence of a steep sea state is not alone sufficient to trigger modulational instability effects. In addition, the wave spectrum needs to be narrow banded in frequency and direction. The relation between the spectral steepness and the spectral bandwidth, here represented by the JONSWAP $\gamma$-like parameter, is shown in Figs. 2 and 3 for wind sea and swell systems, respectively; only sea states with $k_{\mathrm{p}} H_{\mathrm{s}} / 2>0.10$ have been considered. The figures show that a combination of steep and relatively narrow banded sea states for wind sea, representing also the total sea, is not uncommon. However, these conditions were mainly observed with $H_{\mathrm{s}} \approx 3.5 \mathrm{~m}$. For more severe conditions (i.e. $H_{\mathrm{s}}>6 \mathrm{~m}$ and $\left.k_{\mathrm{p}} H_{\mathrm{s}} / 2>0.1\right)$, the JONSWAP $\gamma$-like parameter does not overcome the threshold value of 3 . Approximately $5 \%$ of the records with $H_{\mathrm{s}}>6 \mathrm{~m}$ and $k_{\mathrm{p}} H_{\mathrm{s}} / 2>0.1$ show that the $\gamma$-like parameter oscillates between 2 and 3 . This may be partly related to the procedure applied for estimating the $\gamma$-like parameter; investigations of the accuracy of this specific is, however, outside the scope of the present study. Nonetheless, it is interesting to mention that a JONSWAP spectrum with

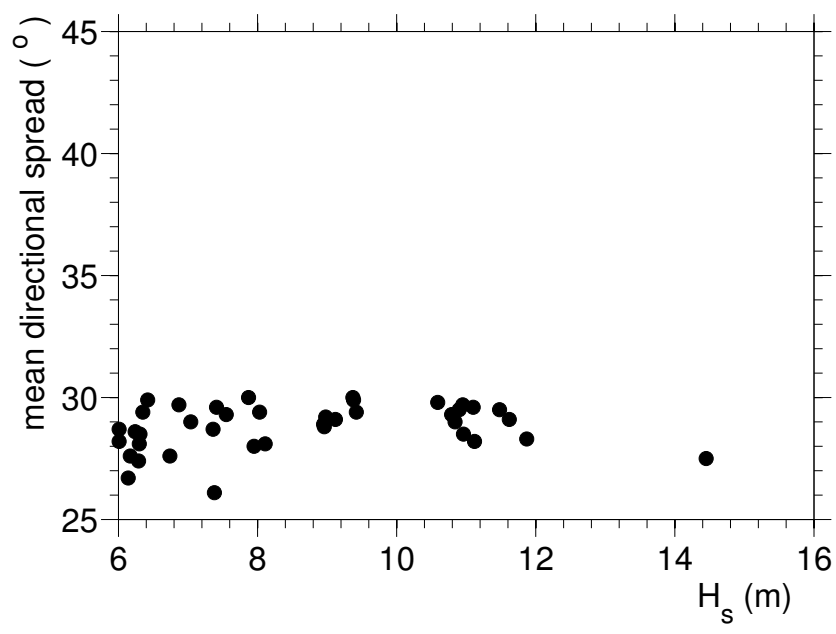

Fig. 4. Mean directional spread for events with $H_{\mathrm{S}}>6 \mathrm{~m}$ and ka $>0.1$ (Oceanweather Inc. hindcast).

$k_{\mathrm{p}} H_{\mathrm{s}} / 2 \approx 0.13$ and $\gamma=2.5$ is characterised by $\mathrm{BFI} \approx 0.9$. Such a condition is sufficient to induce significant deviations from second order model predictions (e.g. Toffoli et al., 2008b).

Apart from steepness and spectral narrowness, also the directional spreading of the wave spectrum plays a fundamental role in dumping (for short crested waves) or enhancing (for long crested waves) effects that are associated to modulational instability. In this respect, we observed that the directional spreading was below 30 degrees for approximately $17 \%$ of the records with $H_{\mathrm{s}}>6 \mathrm{~m}$ and $k_{\mathrm{p}} H_{\mathrm{s}} / 2>0.10$ (see Fig. 4). According to numerical simulations and experimental tests, this condition is likely to lead to moderate deviations from common second order based wave crest distributions (e.g. Socquet-Juglard et al., 2005; Onorato et al., 2009b). However, note that a directional spreading of about 30 degrees has been observed by Waseda et al. (2011) when the rogue events occurred.

Data from the ECMWF reanalysis archive, namely the scatter diagram of significant wave height and spectral period and mean directional spread, are presented in Figs. 5 and 6. Consistently with data from the Oceanweather Inc. hindcast database, relatively large steepness $\left(k_{\mathrm{p}} H_{\mathrm{s}} / 2>0.10\right)$ occurred with similar frequency within the considered 10 yr period. Furthermore, data still show the tendency for the mean directional spread to converge to values of about 30 degrees for the most severe sea states $\left(H_{\mathrm{S}}>10 \mathrm{~m}\right)$.

Based on the hindcast data used in the analysis, it is difficult to specify precisely the duration of the sea states with high $k_{\mathrm{p}} H_{\mathrm{s}} / 2$ and the low mean directional spreading. However, the data indicate that such sea states remained stationary from 3 up to $6 \mathrm{~h}$. Although the modulational instability occurs within 30-40 wavelengths, we can expect that it will 


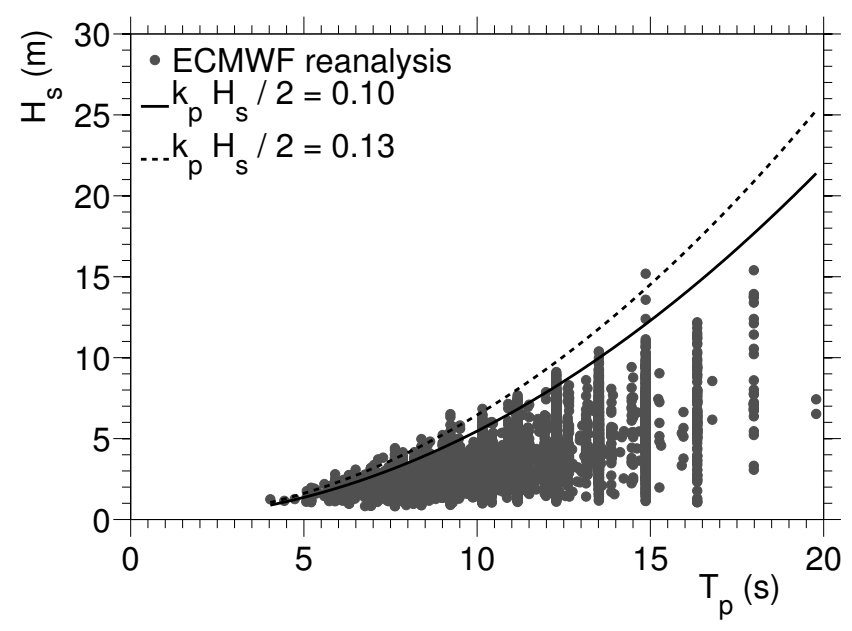

Fig. 5. Scatter diagram of significant wave height $\left(H_{\mathrm{S}}\right)$ and spectral peak period $\left(T_{\mathrm{p}}\right)$ for total seas from ECMWF reanalysis archive; lines of constant spectral steepness $\left(k_{\mathrm{p}} H_{\mathrm{s}} / 2=0.10\right.$ and 0.13$)$ are included.

have larger impact if a wave short-term record is longer (see Sect. 4) .

As aforementioned, ships are design today for the 25-yr return period. Thus, if an event with high steepness and narrow wave directional spread happens once in this period, it needs to be considered in the design process. Further, it should be noticed that the wave ship design data basis provided by IACS (2000) includes sea states with $k_{\mathrm{p}} H_{\mathrm{s}} / 2=0.11-0.13$ and $H_{\mathrm{s}}=16.5 \mathrm{~m}$. However, ship response to extreme events, and not a rogue wave alone, will decide how dangerous for a ship these events will be and whether any revision of ship rules are necessary.

\section{Short-term statistics}

Several studies have been carried out showing short-tem statistics of relevant design wave parameters such as wave crests, wave troughs and wave heights using linear, second order and higher order models (see, for example, Onorato et al., 2009b; Socquet-Juglard et al., 2005; Tayfun and Fedele, 2007; Toffoli et al., 2007, 2008b,a; Toffoli and Bitner-Gregersen, 2011; Waseda et al., 2009). They demonstrate that modulational instability is capable of enhancing the probability of occurrence for extreme events if waves are sufficiently steep, narrow banded and long crested, while its effect is substantially reduced in directional wave fields. In Sect. 3 we have shown that sea states which can trigger modulational instability are not uncommon during the structure's life cycle. However, whether or not extreme wave parameters are observed in a wave record depends upon a sea state duration. In the following, this dependence is demonstrated for wave crest by using experimental and numerical records.

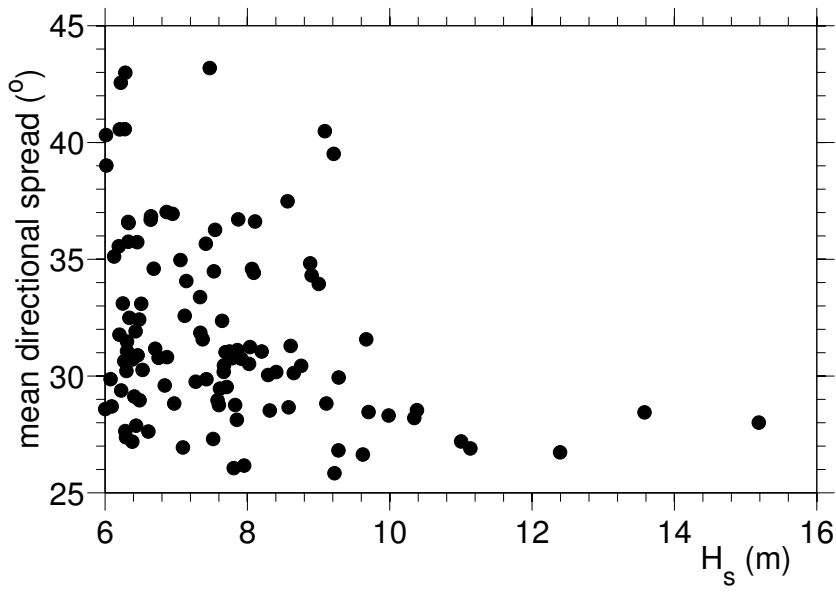

Fig. 6. Mean directional spread for events with $H_{\mathrm{S}}>6 \mathrm{~m}$ and $k_{\mathrm{p}} H_{\mathrm{S}} / 2>0.10$ (ECMWF reanalysis archive).

\subsection{Experiments, numerical simulations and initial conditions}

A set of experimental tests to monitor the nonlinear evolution of random directional wave fields were carried out in the large ocean wave basin at MARINTEK (Norway) and are described in Onorato et al. (2009a). Initial conditions at the wavemaker were generated as a linear superposition of sinusoidal waves from an input directional wave spectrum $E(\omega, \vartheta)=S(\omega) D(\vartheta) . \quad S(\omega)$ has been modelled using the JONSWAP formulation. For convenience, two spectral configurations have been used: one with $T_{\mathrm{p}}=1 \mathrm{~s}$, which corresponds to a dominant wavelength $\lambda_{\mathrm{p}}=1.56 \mathrm{~m}, H_{\mathrm{s}}=0.065$ and $\gamma=3$ and a second with $T_{\mathrm{p}}=1 \mathrm{~s}, H_{\mathrm{s}}=0.080$ and $\gamma=6$. These sea states are characterized by a wave steepness $k_{\mathrm{p}} H_{\mathrm{s}} / 2=0.13$ and 0.16 , respectively (also corresponding to $S_{\mathrm{tp}}=0.041$ and 0.050$)$. Note that the choice of the peak period is arbitrary, and any other wave period representing wind sea could have been applied, provided the same value of steepness is retained. $A \cos ^{N}(\vartheta)$ angular spreading function has been adopted to describe the directional domain $D(\vartheta)$. A number of conditions have been considered, ranging from long crested (large $N$ ) to short crested (small $N$ ) conditions. The following values of the spreading coefficient have been used for the present study: $N=840,90,24$.

Numerical simulations were performed by solving the potential Euler equations with a higher order spectral method (HOSM, West et al., 1987), in order to replicate the laboratory experiments. The initial conditions were therefore identical to the ones adopted in the wave tank. A complete description of the numerical simulations and a detailed comparison against the laboratory measurements is presented in (Toffoli et al., 2008a, 2010). 

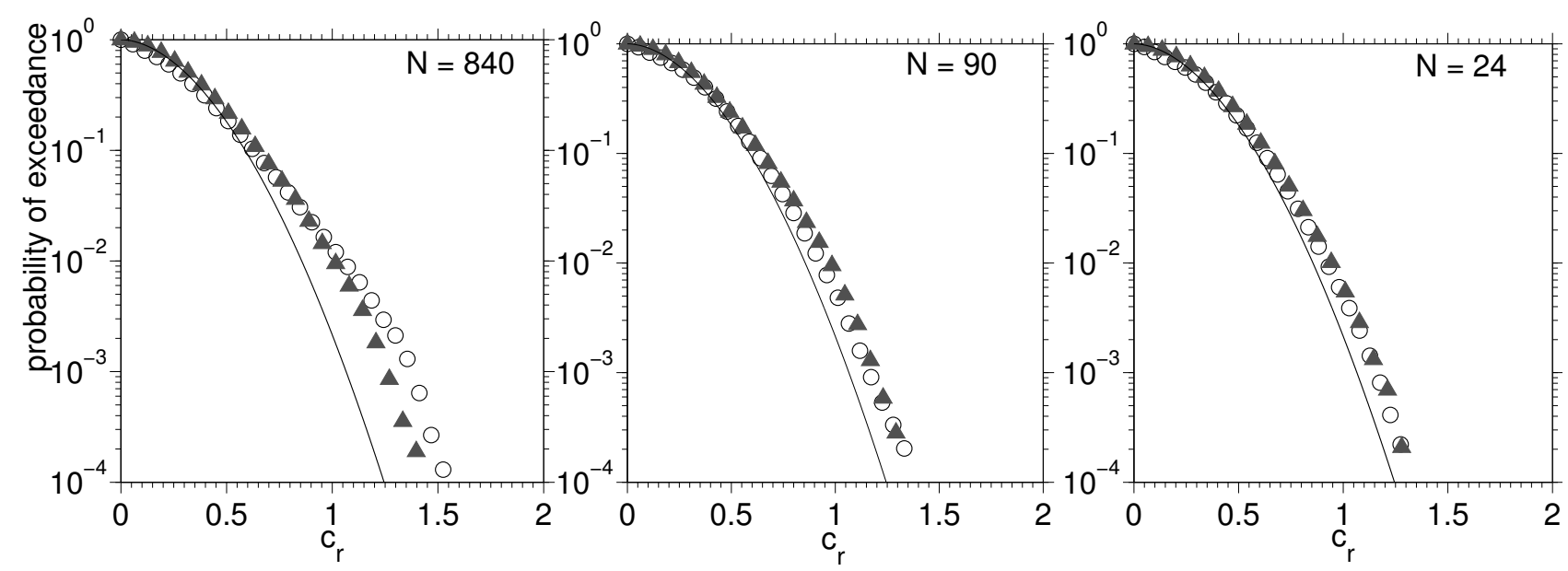

○ Numerical simulations

$\triangle$ Experiments

-Forristall (2000) distr.

Fig. 7. Wave crests distribution: experiments and numerical simulations for a sea states with $k_{\mathrm{p}} H_{\mathrm{s}} / 2=0.16$.

\subsection{Sea state duration and occurrence of rogue wave crests}

An overall analysis and comparison of the statistical properties of experimental and numerical wave data is presented in Toffoli et al. (2010). Here we perform a more detailed statistical analysis of them by fitting the data with a 2-parameter Weibull distribution

$F\left(c_{\mathrm{r}}\right)=1-\exp \left[-\left(\frac{c_{\mathrm{r}}}{\alpha}\right)^{\beta}\right]$

where $\alpha$ is a scale parameter and $\beta$ denotes a slope parameter.

Figure 7 shows the exceedance probabilities $\left(1-F\left(c_{\mathrm{r}}\right)\right)$. The crest statistics based on the numerical and experimental data has coincided well (e.g. see Fig. 7 for example). Some discrepancies are, however, observed for $N=840$ due to the occurrence of wave breaking, which is not accounted for in the numerical model. Considering the agreement between experiments and numerics, only statistics of the experimental data is shown herein.

The fitted Weibull parameters for the selected sea states are reported in Table 1. Although there are no dramatic changes in the Weibull parameters for the different spectral configurations, it is worth mentioning that $\alpha$ and $\beta$ generally increase with the enhancing of wave steepness and decrease with the narrowing of the directional spreading (with larger $N$ ). Modifications induced by these parameters are consistent with the fact that extreme events become more likely for such spectral conditions.

Before using the Weibull parameters for an extreme value analysis, it is important to verify how well the two-parameter Weibull distribution describes the observations. In Fig. 8,
Table 1. Fitted Weibull parameters.

\begin{tabular}{lcc}
\hline Nominal Steepness $k_{\mathrm{p}} H_{\mathrm{s}} / 2=0.13$ & & \\
\hline Directional Spreading $N$ & $\alpha$ & $\beta$ \\
\hline 840 & 0.36 & 1.71 \\
90 & 0.37 & 1.75 \\
24 & 0.37 & 1.77 \\
\hline Nominal Steepness $k_{\mathrm{p}} H_{\mathrm{S}} / 2=0.16$ & & \\
\hline Directional Spreading $N$ & $\alpha$ & $\beta$ \\
\hline 840 & 0.37 & 1.70 \\
90 & 0.38 & 1.76 \\
24 & 0.38 & 1.78 \\
\hline
\end{tabular}

the fitted distribution and the concurrent $95 \%$ confidence intervals (these are calculated based on bootstraps techniques, Emery and Thomson, 2001) are presented against the empirical wave crest distribution for two spectral configurations. On the whole, the fitted Weibull distribution properly described the observations until probabilities as low as $10^{-3}$. For lower probabilities, however, a deviation from the fitted distribution is observed. Although this is expected due to uncertainties related to the limited number of observations at low probability levels (this is, in fact, shown by the broadening of the confidence intervals), this deviation is more pronounced for narrow directional spreading conditions; the observations remains within the $95 \%$ confidence limits though. Note that the 2-parameter Weibull distribution seems to overestimate slightly the wave crests for low probability levels. 


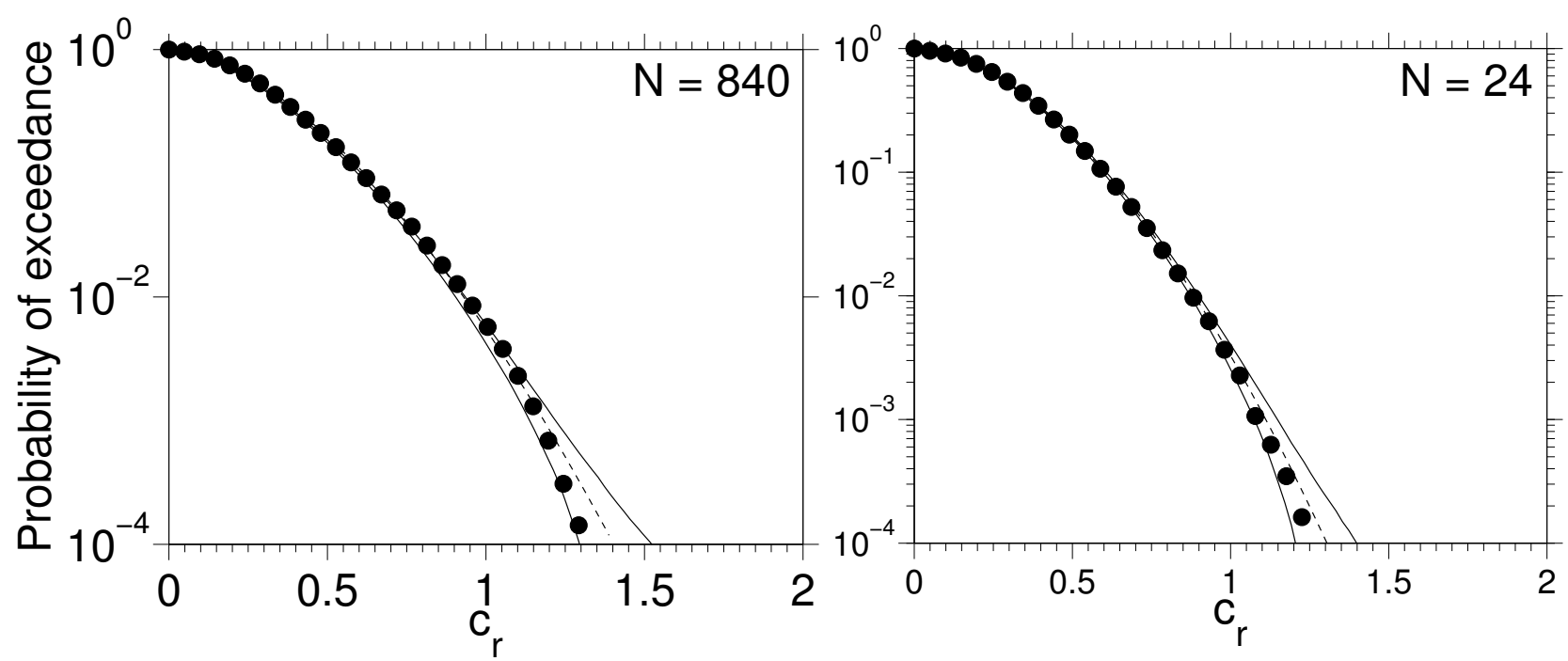

Fig. 8. Weibull fit (dashed line) and $95 \%$ confidence intervals (solid line) for the wave crest distribution (circles) associated to a nominal sea state with $k_{\mathrm{p}} H_{\mathrm{s}} / 2=0.16$.

Verified that the two-parameter Weibull fit $F\left(c_{\mathrm{r}}\right)$ is acceptable approximation of the wave crest distribution and assumed that wave crests are independent events, the extreme short-term crest distribution $F_{\mathrm{ES}}\left(c_{\mathrm{r}}\right)$ for a specified sea state duration $(\tau)$ reads (see, e.g. Gumbel, 1958)

$F_{\mathrm{ES}}\left(c_{\mathrm{r}}\right)=\left\{1-\exp \left[-\left(\frac{c_{\mathrm{r}}}{\alpha}\right)^{\beta}\right]\right\}^{n}$,

where $n$ denotes the number of expected wave crest outcomes in a $\tau$-hour time period.

Often, specific features of the extreme non-dimensional crest distribution (3) are of interest in design applications such as the mean, the median or the characteristic largest value (hereafter referred to as $c_{\mathrm{c}}$ ). The latter can be calculated from the 2-parameter Weibull distribution (2) as the $\tau$ hour return value as follows (Gumbel, 1958):

$F\left(c_{\mathrm{c}}\right)=1-\frac{1}{n}$

or, for large $n$, equivalently derived from Eq. (3) as

$F_{\mathrm{ES}}\left(c_{\mathrm{c}}\right)=F\left(c_{\mathrm{c}}\right)^{n}=\left(1-\frac{1}{n}\right)^{n} \approx \frac{1}{e}$.

Note that, as for a large number of crest observations, the distribution in Eq. (3) becomes approximately the Gumbel distribution.

More convenient, particularly for applications, is to express the characteristic largest value of the non-dimensional wave crest as defined by Eq. (4) by the Weibull parameters $\alpha$ and $\beta$, of the distribution (2):

$c_{\mathrm{c}}=\alpha[\ln (n)]^{1 / \beta}$.
Note that the median can also be expressed by the Weibull parameters

$c_{\mathrm{m}}=\alpha\left[-\ln \left(1-0.5^{1 / n}\right)\right]^{1 / \beta}$.

We stress the fact that the extreme values given by Eqs. (6) and (7) depend on the assumed sea state duration $(\tau)$ through the number of outcomes $n$.

Figures 9 and 10 present the characteristic largest crest $c_{\mathrm{c}}$, (derived according to Eq. 5) in a wave record normalised by the significant wave height as a function of sea state duration. It is evident that the extreme crest considerably increases for prolonged sea state durations. In order to capture individual waves with $c_{\mathrm{c}}>1.4$ (i.e. $c_{\max } / H_{\mathrm{s}}>1.4$, where $c_{\max }$ is the absolute characteristic largest value) in particular, a sea state duration of about $5 \mathrm{~h}$ is needed for $k_{\mathrm{p}} H_{\mathrm{s}} / 2=0.13$ and long-crested waves $(N=840)$, while only $3 \mathrm{~h}$ are sufficient for $k_{\mathrm{p}} H_{\mathrm{s}} / 2=0.16$ and long-crested waves. The second order model, however, is not capable to capture such an event even if a $9 \mathrm{~h}$ duration is considered. Furthermore, the figures indicate that the wave directional spreading has significant impact on extreme crest statistics. Note that the effect of directional spreading is more pronounced for $k_{\mathrm{p}} H_{\mathrm{s}} / 2=0.16$ than for $k_{\mathrm{p}} H_{\mathrm{s}} / 2=0.13$ and increases with increase sea state duration.

For design purposes a sea state duration of 3 or $6 \mathrm{~h}$ is usually applied (DNV, 2010). In this respect, the results indicate that wave crests with $c_{\mathrm{c}}>1.4$ can actually be observed if a rogue-wave-prone sea state remains stationary for the aforementioned durations and waves are sufficiently long-crested. Such an event still remains likely also for broader directional sea states, provided sea state duration is longer than $6 \mathrm{~h}$. 


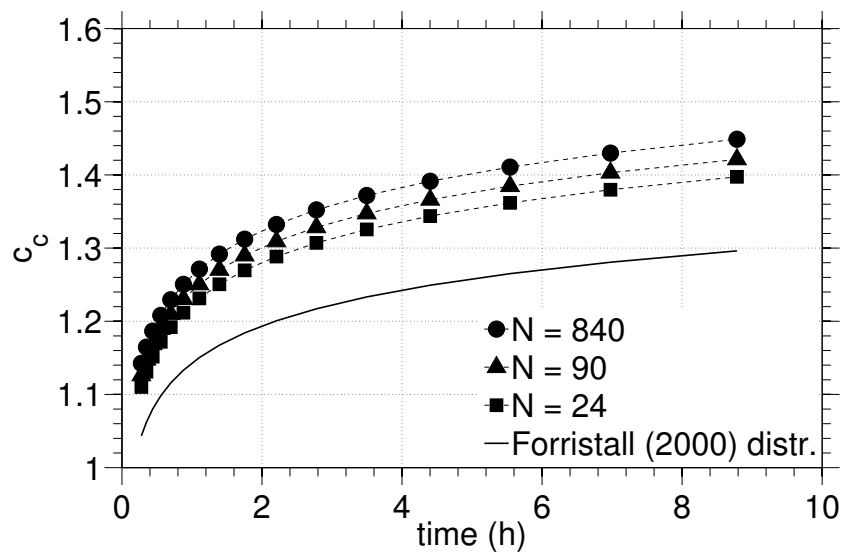

Fig. 9. Wave crest probability as a function of the sea state duration for a nominal sea state with $k_{\mathrm{p}} H_{\mathrm{s}} / 2=0.13$ (experiments). $c_{\mathrm{c}}$ here represents the characteristic largest value for the non-dimensional wave crest $c_{\mathrm{r}}$.

\subsection{Uncertainty of the results}

The results shown in Figs. 9 and 10 are affected by several types of uncertainties such as data uncertainty due to accuracy of laboratory measurements as well as the method adopted for generating waves, statistical uncertainty due to a limit number of observations in a wave record, and model uncertainty due to the model applied to fit the data. Specification of these uncertainties as well as their impact on the probability of occurrence of extreme and rogue crests are of importance for design. To investigate this, a simplified model has been adopted and the probability of occurrence of a rogue wave event in a random sea state of a given sea state duration is calculated

$P\left(c_{\mathrm{r}}>\zeta\right)=P\left[\alpha_{N}, \eta^{(2)}\left(H_{\mathrm{s}}, T_{\mathrm{Z}}, d\right)>c_{e}\right]$,

where $\zeta$ denotes a given ratio $c / H_{\mathrm{s}}, c_{e}=\zeta H_{\mathrm{s}}$ is a rogue wave crest, $\eta^{(2)}$ is the second order undisturbed (without a marine structure) local surface elevation (extreme crest for a given sea state duration) as given by the Forristall (2000) distribution, $\alpha_{N}$ is a correction factor due to higher order effects (includes data and model uncertainty), $T_{\mathrm{Z}}$ denotes the zerocrossing wave period, while $d$ is the water depth.

Using the Structure Reliability Analysis (SRA), the probability of extreme crest has been calculated by FORM and SORM (see, e.g. Madsen et al., 1986 for details). FORM (First Order Reliability Method) represents a linear while SORM (Second Order Reliability Method) a quadratic approximate method for calculating probabilities. The reliability methodology allows for consistent treatment of uncertainties and provides probabilities where uncertainties can be included. By adopting these structural reliability methods, sensitivity studies can be carried out and importance of analysed

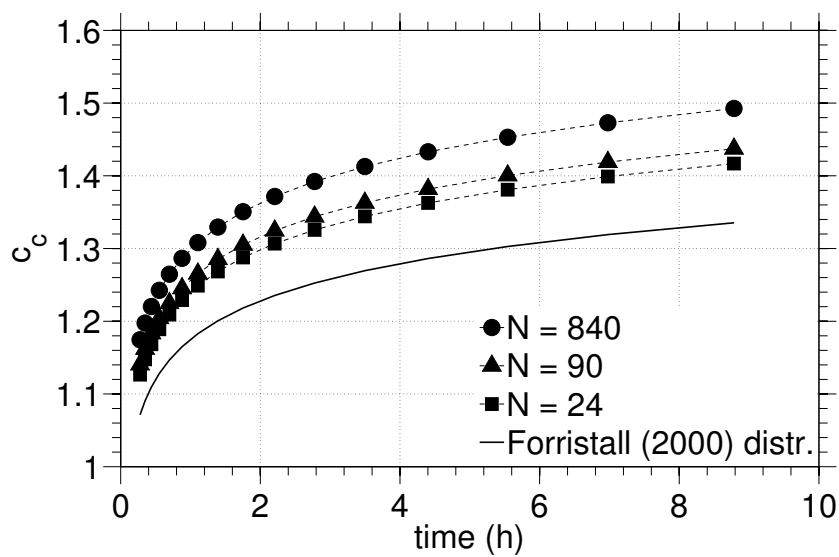

Fig. 10. Wave crest probability as a function of the sea state duration for a nominal sea state with $k_{\mathrm{p}} H_{\mathrm{s}} / 2=0.16$ (experiments). $c_{\mathrm{c}}$ here represents the characteristic largest value for the non-dimensional wave crest $c_{\mathrm{r}}$.

parameters to the failure probability (crest reaching a given threshold) identified.

The Uncertainty Importance Factor is defined as the square of the geometrical sensitivity factor (see Hohenbichler and Rackwitz, 1986, for details). It indicates the importance of modelling the random variable $X_{i}$ as a distributed variable rather than as a fixed valued variable, the median of the distribution being the fixed value. In other words, the Uncertainty Importance Factor of the $i$-th variable roughly gives the fraction of the total uncertainty that is caused by uncertainty in this variable.

The following uncertainties have been included in the analysis when calculating the probability of crest reaching a given threshold: uncertainty of the correction factor for nonlinearities and sampling variability of $H_{\mathrm{s}}$ and $T_{\mathrm{Z}} . \alpha_{N}$ has been assumed to be normally distributed, with the mean value equal 1.01-1.20 and coefficient of variation $\mathrm{COV}=0.02-0.10(\mathrm{COV}=\mathrm{std} / \mathrm{mean})$ for the 2-D sea state, and the mean value equal $1.05-1.10$ and $\mathrm{COV}=0.05$ for $3-\mathrm{D}$ waves, respectively. These numbers have been adopted based on examinations of experimental and numerical data. The sampling variability has been adopted as theoretically derived by Bitner-Gregersen and Hagen (1990) $\left(H_{\mathrm{S}}\right.$ and $T_{\mathrm{Z}}$ normally distributed with $\mathrm{COV}=0.054$ for $H_{\mathrm{S}}$ and $\mathrm{COV}=0.028$ for $T_{\mathrm{Z}}$ ).

The importance factors for a long crested case are shown in Table 2. The results referred to $c_{\max } / H_{\mathrm{s}}>1.4,\left(c_{e}=\right.$ $\left.1.4 H_{\mathrm{S}}\right), \alpha_{N}=1.11(\mathrm{COV}=0.02$ has been adopted $)$ and the $3 \mathrm{~h}$ sea state duration (see Fig. 10). As expected, the adopted second order crest distribution has the largest effect on the probability of $c_{\max } / H_{\mathrm{s}}>1.4(68 \%)$, the sampling variability of $H_{\mathrm{S}}$ contributes $28 \%$ while the inaccuracy of $\alpha_{N}$ and $T_{\mathrm{p}} 3 \%$ and $1 \%$, respectively. Note that the low $\mathrm{COV}$ for the 
Table 2. Importance factors for $c_{\max } / H_{\mathrm{S}}>1.4$.

\begin{tabular}{lc}
\hline $\begin{array}{l}\text { Random } \\
\text { variable }\end{array}$ & $\begin{array}{c}\text { Importance } \\
\text { factor }\end{array}$ \\
\hline$\eta^{(2)}$ & $68 \%$ \\
$H_{\mathrm{S}}$ & $28 \%$ \\
$\alpha_{N}$ & $3 \%$ \\
$T_{\mathrm{Z}}$ & $1 \%$ \\
\hline
\end{tabular}

nonlinear correction factor has been introduced. For higher values of COV the importance of uncertainty of this factor increases to above $10 \%$. The same trends have been observed for others $c_{\mathrm{max}} / H_{\mathrm{s}}$ ratios as well as for directional spread waves. This means that the uncertainty introduced into the analysis by $H_{\mathrm{s}}$ is essential for the final results. The finding is of importance when using the simulation results in the design process and should be kept in mind when carrying out model tests as well as numerical calculations.

\section{Conclusions}

Generally speaking, when a definition of rogue waves and their probability of occurrence have to be provided, distinctions need to be made whether or not a structure is involved, as the type of structure as well as its response will decide the dangerousness of the rogue wave. The present study is limited to rogue waves only and hence it does not cover interaction between rogue waves and marine structures. For the latter, reference is made to the EXTREME SEAS project.

Under these circumstances, it is nowadays well established that sea states characterised by high steepness and narrow directional distribution are prone to modulational instability mechanisms and hence the formation of rogue waves. Here we used hindcast data to investigate the frequency of occurrence of such sea states. Results reveal that rogue-waveprone sea states can actually occur more often than once in the 25-yr period, which is currently used as a return period for ship design. The investigations carried out by Bascheck and Imai (2011) support this conclusion. It is important to note, however, that hindcast data are generally subjected to a number of uncertainties, which may have influenced the present result. Nonetheless, investigation of wave model uncertainties was outside the scope of the present study.

Based on rogue-wave-prone sea states, the importance of the sea state duration and uncertainties involved in probability of occurrence of individual extreme and rogue crests in a wave record is addressed. In particular, the results of the study suggest that sea state duration has a substantial effect on the value of the extreme crest observed in a wave record both for long-crested as well as directionally spread waves. Within a typical design sea state duration of 3 to $6 \mathrm{~h}$, individual waves with $c_{\max } / H_{\mathrm{s}}>1.4$ are likely for long crested sea states, while longer durations may be needed to observe these waves for broader directional sea states. It should be mentioned also, that evaluation of accuracy of significant wave height used in numerical and experimental simulations is essential for providing a good estimate of probability of extreme crest occurrence.

Further investigations of met-ocean conditions in which rogue waves occur are still needed to reach firm conclusions about probability of occurrence of rogue waves. Studies based on instrumental directional and spatial wave data are particularly called for.

Acknowledgements. This research has been supported by EU project EXTREME SEAS (contract SCP8-GA-2009-234175) and the Australian Research Council and Woodside Energy Ltd Linkage project LP0883888. Experiments were supported by the European Community's Sixth Framework Programme through the grant to the budget of the Integrated Infrastructure Initiative HYDRALAB III within the Transnational Access Activities, Contract no. 022441. A. T. also acknowledge support from the Research Development Scheme of Swinburne University of Technology. Authors thanks Shell International Exploration and Production B. V. and Meteo France for providing hindcast data. M. Onorato and M. Benoit are acknowledged for discussions.

Edited by: I. Didenkulova

Reviewed by: P. Liu and another anonymous referee

\section{References}

Bascheck, B. and Imai, J.: Rogue Wave Observations off the US West Coast, Oceanography, 24, 158-165, doi:10.5670/oceanog.2011.35, 2011.

Bitner-Gregersen, E.: Sea state duration and probability of occurrence of a freak wave, in: Proc. 22nd International Conference on Offshore Mechanics and Arctic Engineering (OMAE), Cancun, Mexico, 2003.

Bitner-Gregersen, E.: Joint probabilistic description for combined seas, in: Proc. 24th Int. Conf. Offshore Mechanics and Artic Eng. (OMAE), Halkidiki, Greece, 2005.

Bitner-Gregersen, E. M.: Joint long-term models of met-ocean parameters, in: CENTEC Anniversary Book, in press, A.A. Balkema Publishers, Taylor and Francis, The Netherlands, 2012.

Bitner-Gregersen, E. M. and de Valk, C.: Quality control issues in estimating wave climate from hindcast and satellite data, in: Proc. 27th Int. Conf. Offshore Mechanics and Arctic Eng. (OMAE), Estoril, Portugal, oMAE2008-57865, 2011.

Bitner-Gregersen, E. M. and Guedes Soares, C.: Uncertainty of Average Wave Steepness Prediction from Global Wave Databases, in: Advancements in Marine Structures, edited by: Guedes Soares, C. and Das, P. K., Taylor and Francis Group, London, UK, 3-10, 2007.

Bitner-Gregersen, E. M. and Hagen, Ø.: Uncertainties in Data for the Offshore Environment, Struct. Safety, 7, 11-34, doi:10.1016/0167-4730(90)90010-M, 1990.

Bitner-Gregersen, E. M. and Hagen, $\varnothing$.: Freak wave events within the second order wave model, in: Proc. 23th Int. Conf. on Off- 
shore Mechanics and Arctic Engineering (OMAE), Vancouver, Canada, 2004.

Christos, M. and Ewans, K.: Examining a Comprehensive Dataset Containing Thousands of Freak Wave Events. Part 1 - Description of the Data and Quality Control Procedure, in: Proc. 30th Int. Conf. Offshore Mechanics and Artic Eng. (OMAE), Rotterdam, the Netherlands, oMAE2011-50168, 2011a.

Christos, M. and Ewans, K.: Examining a Comprehensive Dataset Containing Thousands of Freak Wave Events. Part 2 - Analysis and Findings, in: Proc. 30th Int. Conf. Offshore Mechanics and Arctic Eng. (OMAE), Rotterdam, the Netherlands, oMAE201150169, $2011 \mathrm{~b}$.

DNV: DNV RP-C205: Environmental Conditions and Environmental Loads, Tech. rep., Det Norske Veritas, DNV, Høvik, Norway, 2010.

Dysthe, K., Krogstad, H. E., and Müller, P.: Oceanic rogue waves, Annu. Rev. Fluid Mech., 40, 287-310, doi:10.1146/annurev.fluid.40.111406.102203, 2008.

Emery, W. and Thomson, R.: Data Analysis Methods in Physical Oceanography, Advanced Series on Ocean Engineering - Vol. 2, Elsevier Science B.V., Amsterdam, 2001.

Forristall, G.: Wave crests distributions: observations and secondorder theory, J. Phys. Ocean., 30, 1931-1943, 2000.

Gramstad, O. and Trulsen, K.: Influence of crest and group length on the occurrence of freak waves, J. Fluid Mech., 582, 463-472, 2007.

Guedes Soares, C., Cherneva, Z., and Antão, E.: Abnormal waves during Hurricane Camille, J. Geophys. Res., 109, C08008, doi:10.1029/2003JC002244, 2004.

Gumbel, E. J.: Statistics of extremes, Columbia Univ. Press, New York, 1958.

Hauser, D., Kahma, K. K., Krogstad, H. E., Lehner, S., Monbaliu, J., and Wyatt, L. W. (Eds.): Measuring and analysing the directional spectrum of ocean waves, Cost Office, Brussels, 2005.

Haver, S. and Andersen, J.: Freak waves: rare realizations of a typical population or typical realizations of a rare population?, in: Proc. 10-th Int. Offshore and Polar Engineering (ISOPE) Conf., Seattle, USA, 2000.

Haver, S. and Winterstein, S. R.: Environmental contour lines: A method for estimating long term extremes by a short term analysis, Transactions of the Society of Naval Architects and Marine Engineers, 116, 116-127, 2009.

Hohenbichler, M. and Rackwitz, R.: Sensitivity and importance measures in structural reliability, Civil Eng. Syst., 3, 203-209, 1986.

IACS: IACS Rec 34 Standard Wave Data, Tech. Rep. Rev. 1, Corr., IACS, 2000.

IMO: Interim Guidelines for the Application of Formal Safety Assessment (FSA) to the IMO Rule Making Process, Tech. rep., Maritime Safety Committee, 68th session, June 1997; and Marine Environment Protection Committee, 40th session, September 1997, 1997.

IMO: Guidelines for Formal Safety Assessment for the IMO Rule Making Process, Tech. rep., IMO/Marine Safety Committee 74/WP.19, 2001

Janssen, P. A. E. M.: Nonlinear four-wave interaction and freak waves, J. Phys. Ocean., 33, 863-884, 2003.

Kharif, C. and Pelinovsky, E.: Physical mechanisms of the rogue wave phenomenon, Eur. J. Mech. B/Fluids, 22, 603-634, 2003.
Kharif, C., Pelinovsky, E., and Slunyaev, A.: Rogue Waves in the Ocean, Advances in Geophysical and Environmental Mechanics and Mathematics, Springer, Berlin, 2009.

Loffredo, L., Monbaliu, J., Bitner-Gregersen, E. M., and Toffoli, A.: The role of spectral multimodality in wave climate design, in: 11th International Workshop on Wave Hindcasting and Forecasting, Halifax, NS, Canada, 2009.

Madsen, H. O., Krenk, S., and Lind, N. C.: Methods of Structural Safety, Prentice-Hall, Enlewood Cliffs, NJ, 1986.

Mori, N. and Janssen, P. A. E. M.: On kurtosis and occurrence probability of freak waves, J. Phys. Ocean., 36, 1471-1483, 2006.

Mori, N., Liu, P. C., and Yasuda, T.: Analysis of freak wave measurements in the Sea of Japan, Ocean Eng., 29, 1399-1414, 2002.

Mori, N., Onorato, M., and Janssen, P.: On the estimation of the kurtosis in directional sea states for freak wave forecasting, J. Phys. Ocean., 41, 1484-1497, 2011.

NORSOK: Standard N-003: Action and action effects, Norwegian Technology Standardization, Oslo, Norway, 2007.

Olagnon, M.: About the frequency of occurrence of rogue waves, in: Proceeding of Rogue Waves 2008 Workshop, Brest, France, 2008.

Olagnon, M. and Magnusson, A. K.: Sensitivity study of sea state parameters in correlation to extreme wave occurrences, in: Proc. 14th International Offshore and Polar Engineering Conference (ISOPE), Toulon, France, 2004.

Olagnon, M. and Prevosto, M. (Eds.): Proceedings of the Rogue Waves 2008 Workshop, IFREMER, Brest, 2008.

Onorato, M., Osborne, A. R., Serio, M., and Cavaleri, L.: Modulational instability and non-Gaussian statistics in experimental random water-wave trains, Phys. Fluids, 17, 078101, doi:10.1063/1.1946769, 2005.

Onorato, M., Osborne, A., Serio, M., Cavaleri, L., Brandini, C., and Stansberg, C.: Extreme waves, modulational instability and second order theory: wave flume experiments on irregular waves, Europ. J. Mech. B/Fluids, 25, 586-601, 2006.

Onorato, M., Cavaleri, L., Fouques, S., Gramstad, O., Janssen, P. A. E. M., Monbaliu, J., Osborne, A. R., Pakozdi, C., Serio, M., Stansberg, C., Toffoli, A., and Trulsen, K.: Statistical properties of mechanically generated surface gravity waves: a laboratory experiment in a 3D wave basin, J. Fluid Mech., 627, 235-257, 2009a.

Onorato, M., Waseda, T., Toffoli, A., Cavaleri, L., Gramstad, O., Janssen, P. A. E. M., Kinoshita, T., Monbaliu, J., Mori, N., Osborne, A. R., Serio, M., Stansberg, C., Tamura, H., and Trulsen, K.: Statistical properties of directional ocean waves: the role of the modulational instability in the formation of extreme events, Phys. Rev. Lett., 102, 114502, doi:10.1103/PhysRevLett.102.114502, 2009b.

Osborne, A. R.: Nonlinear Ocean Waves and the Inverse Scattering Transform, International Geophysics Series, Elsevier, San Diego, 2010.

Rosenthal, W. and Lehner, S.: Rogue Waves: Results of the MaxWave Project, J. Offshore Mech. Arct. Eng., 130, 021006, doi:10.1115/1.2918126, 2008.

Skjong, R. and Bitner-Gregersen, E. M.: Cost Effectiveness of Hull Girder Safety, in: Proc. 21st Int. Conf. Offshore Mechanics and Artic Eng. (OMAE), Oslo, Norway, 2002.

Socquet-Juglard, H., Dysthe, K., Trulsen, K., Krogstad, H., and Liu, J.: Distribution of surface gravity waves during spectral changes, 
J. Fluid Mech., 542, 195-216, 2005.

Tamura, H., Waseda, T., and Miyazawa, Y.: Freakish sea state and swell-windsea coupling: Numerical study of the Suwa-Maru incident, Geophys. Res. Lett., 36, L01607, doi:10.1029/2008GL036280, 2009.

Tayfun, M. A. and Fedele, F.: Wave-height distributions and nonlinear effects, Ocean Eng., 34, 1631-1649, 2007.

Toffoli, A. and Bitner-Gregersen, E. M.: Extreme and Rogue Waves in Directional Wave Fields, Open Ocean Eng. J., 4, 24-33, doi:10.2174/1874835X01104010024, 2011.

Toffoli, A., Onorato, M., Babanin, A. V., Bitner-Gregersen, E., Osborne, A. R., and Monbaliu, J.: Second-order theory and set-up in surface gravity waves: a comparison with experimental data, J. Phys. Ocean., 37, 2726-2739, 2007.

Toffoli, A., Bitner-Gregersen, E., Onorato, M., and Babanin, A. V.: Wave crest and trough distributions in a broad-banded directional wave field, Ocean Eng., 35, 1784-1792, 2008 a.

Toffoli, A., Onorato, M., Bitner-Gregersen, E., Osborne, A. R., and Babanin, A. V.: Surface gravity waves from direct numerical simulations of the Euler equations: a comparison with secondorder theory, Ocean Eng., 35, 367-379, 2008b.
Toffoli, A., Gramstad, O., Trulsen, K., Monbaliu, J., BitnerGregersen, E. M., and Onorato, M.: Evolution of weakly nonlinear random directional waves: laboratory experiments and numerical simulations, J. Fluid Mech., 664, 313-336, doi:10.1017/S002211201000385X, 2010.

Tomita, H. and Kawamura, T.: Statistical Analysis and Inference from the In-Situ Data of the Sea of Japan with Reference to Abnormal and/or Freak Waves, in: Proc. 10th International Offshore and Polar Engineering Conference, Seattle, USA, 2000.

Waseda, T., Kinoshita, T., and Tamura, H.: Evolution of a random directional wave and freak wave occurrence, J. Phys. Oceanogr., 39, 621-639, 2009.

Waseda, T., Hallerstig, M., Ozaki, K., and Tomita, H.: Enhanced freak wave occurrence with narrow directional spectrum in the North Sea, Geophys. Res. Lett., 38, doi:10.1029/2011GL047779, 2011.

West, B. J., Brueckner, K. A., Jand, R. S., Milder, D. M., and Milton, R. L.: A new method for surface hydrodynamics, J. Geophys. Res., 92, 11803-11824, 1987.

Zakharov, V. and Ostrovsky, L.: Modulation instability: The beginning, Physica D, 238, 540-548, 2009. 